\title{
The Effect of Bisphenol A on the Histological Parameters of Male Rat Prefrontal Area
}

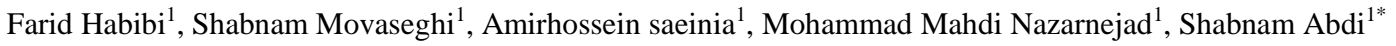 \\ 1- Department of Anatomical Sciences \&Cognitive Neuroscience, Faculty of Medicine, Tehran Medical Sciences, Islamic Azad University, Tehran, Iran.
}

\section{A B S T R A C T}

Background and Objectives: Bisphenol-A (BPA) is a substance used in epoxy resin monomers and polycarbonate plastics. This research focuses on the effect of Bisphenol A on histology alterations in prefrontal cortex (PFC) of rats.

Materials and Methods: Thirty male rats were divided into 3 groups: A control group, a placebo group received distilled water intra peritoneal and the experiment group received $1.0 \mu \mathrm{g} / \mathrm{kg}$ BPA intra peritoneal for 14 days. After 2 weeks, rat's brains were enucleated, sequenced in $10 \mu \mathrm{m}$ widths and $\mathrm{HE}$ stained for histological examinations. Neural and neuroglia cells were counted and rats' PFC volume was measured using stereological methods.

Results: Our results showed statistically significant decreases in PFC volume and neural cell count in the experiment group in comparison to both placebo and control groups. Also, results showed statistically significant increases in glial cell count in the experiment group in comparison to other groups.

Conclusions: This research showed that BPA has negative and pathological effects on neural cells and PFC in rats

Keywords: Bisphenol A, Prefrontal cortex, Rats

\section{Introduction}

Nowadays thousands of various chemical and industrial substance enter an environment which is harmful and disruptive of the balance of human and other animal endocrine system, one of which Bisphenol-A (BPA) can be mentioned(1-3) (4). Since BPA is lipophilic and widely used in producing medical equipment and disposal utensils, it can penetrate to the body by mucous membrane and skin contact. BPA is $(5,6)$. This environmental pollutant can enter the human food chain through air, water and food. BPA is diagnosed in $93 \%$ of American's urinary tract which shows that it enters human body easily and in an enormous amount. BPA can interfere with the synthesis and clearance of hormones and it can change gene expressions and the gene function of target tissue $(7,8)$. Also, studies on Laboratory animals determined that BPA has harmful effects on the brain, reproduction system, metabolic processes that are involved in insulin's hemostasis and liver enzymes. Several types of research show that BPA prevents the development of synapses depended on steroid gonad hormones in hippocampus and prefrontal cortex in male and female mouse and primates except for humans. Based on a literature review BPA by changing the gene expression pattern in central nervous system cells and also producing Reactive Oxygen Species
(ROS) can cause a reduction of endothelial MSS 31 class ability or even their death (9-11).

Prefrontal cortex of the brain is involved in complicated perceptual behaviors of brain, personality expression, decision making and managing the right social behaviors. This area of brain is responsible for high level cognitive activity and executive function. Persons suffering from neural development disorder in some of these areas show low activity which might express their defection in social understanding and comprehension. Also, damages caused to these areas create symptoms of autism for animals and social cognition challenges for humans $(12,13)$. Regarding the mentioned issues and despite all of the studies done in the neural development disorder field and the treatments related to it, there are still lots of unanswered questions or there isn't an accurate and specific answer for them. The influences of BPA on the structure on the brain especially on the inner layer of prefrontal cortex area is more concentrated on behaviour tests right now. This approach of studying is not suitable so an approach is needed to aim to study the tissues in three-dimensional form. That is why scientists examined 3D structural study method which are called stereology. For this very reason, in this study the 
possibility of histological changes in the prefrontal area of male rats after sudden exposure of BPA was examined.

\section{Materials and Methods}

\section{Animals and Dosing Procedure}

In this study 30 male rats from wistar race with a range of weight between 200-250 (gr) were purchased from the laboratory animal center of Pasteur Institute, Tehran, Iran. Animals were maintained in a suitable condition with 12 hours of brightness and 12 hours of darkness, temperature between 20-24 ${ }^{\circ} \mathrm{C}$ and 40-50 percent humidity. Enough standard laboratory rodent food and water were available for them. They were randomly arranged in three groups. The first group didn't receive any treatment, for second group or placebo group normal saline was injected intraperitoneally, to the third group or test group 100 microgram per kilogram dose of BPA by body weight was injected.

\section{Bisphenol-A treatment}

Chemical substances and experimental group of BPA which were white and solid powder form was provided from Merck KGaA German corporate. Distilled water was used as solvent and BPA was injected intraperitoneally with a dosage of 100 micrograms per kilogram in the length of two weeks to rats. All rats were kept for 14 days after the BPA injection, and then the prefrontal was analysing.

\section{Histology}

After two weeks, rats were killed and after tissue fixation, tissue circulation and paraffin blocks sample were prepared. Incisions thickness was 10 microns and they were stained with karzil violet and hematoxylin and eosin method. The incisions from prefrontal cortex area were obtained based on stereological laws from the Isotropic uniform random type. For determining the volume of prefrontal cortex area cavalieri's method and for counting the numbers of neurons and neuroglia optical dissector method and microcator device were used. From all of the groups tissue samples were obtained, these samples immediately after perfusion were placed in $4 \%$ buffered fixative solvent so that they become fixed. After 14 days of BPA injection perfusion was done, first with a weighing device the weight of rats was obtained, then they passed out, after that they were lied down from posterior region and their hands and legs were tied to surgical bed. Also the surgical bed was inclined so that the head of rat would face downwardly and more fluid flew into the rat's brain. With a cut lower from sternum xiphoid process with 1 centimetre depth, chest was unknited and heart was separated from pericardium. By pulling away left lung, perfusion was injected from the top of the heart to the left ventricle. By stabilizing low rate flow on normal saline to left ventricle, right atrium was splashed so the blood came out. 200-250 cc physiological serum was injected to animal vessels for clearing. This action continued until animal hands and legs became white and the fluid that was coming out of the animal right atrium became clarified. After saline wash, the same connective set with 200-250 cc $10 \%$ formalin fixative was injected to animal's vessels.. For fixation of tissue, samples were placed in $4 \%$ formaldehyde fixative solvent for 72 hours. For dehydration, samples were transferred for 2-4 hours into 5 containers which by order had \%70, \%80, $\% 96$, and two \%100 alcohol. For clearing, three container containing Xylenol was used. Each sample was placed into these containers for 3 to 6 hours. For infiltration, solid paraffin which was pure and clear was used. This paraffin was melted in $56-60{ }^{\circ} \mathrm{C}$ temperature and then sample was placed in paraffin which its volume was 20-30 times more than the sample. This action consecutively occurred 3 times from container to container and with a 3-6 hour length. For embedding, initially a little melted paraffin was poured on the floor of template, then the intended tissue was grabbed by pence and was folded into the same paraffin and again paraffin was poured on them. Also the information of tissues which was written on paper with pencil was folded at the bottom/end of paraffin. After 30 minutes paraffin cooled, template was turned over so that the paraphrased tissue gets separated from template. At the end of this action, templates were folded in room temperature. After preparing paraphrased blocks of rat's prefrontal cortex, coronal sectioning was done by the assessment of rotary microtome in 10 microne thickness.

\section{Cavalieri's technique}

Stereology examining method is used for studying the slides of histology. In this method an estimation of the volume of prefrontal cortex and an estimation of number of neurons and neuroglia was made. Determining the volume of prefrontal cortex was done by cavalieri's technique. Estimating the number of neurons and neuroglia was done by optical dissector method (14). In cavalieri's technique, first the tissue was sectioned with serial sectioning method. In next step, 10 tissue sections were randomly choosing for this technique. Initially all of the sections of one sample was divided by 10 , the first tissue section was randomly selected at a distance of 0-10, and the next section was selected at a distance from the previous section. Thus, 10 section from 1 sample was obtained. Then, a multipurpose test system which had 100 dots for calculating the volume of prefrontal cortex was used. In this network, dots were randomly placed on tissue sections by counting the number of dots, the volume of nucleus was calculated with a formula which was $V=\Sigma p \times a /(p \times t)$. In this study, projecting microscope with magnification of 40 was utilized and total volume was mentioned in millimetre dimension. 
The fixed number in this formula is: $\Sigma p$ which shows the number of dots from test system which were placed on tissue. For calculating a/p another formula was used which was $\mathrm{a} / \mathrm{p}=\Delta \mathrm{y} \times \Delta \mathrm{x} / \mathrm{M}^{\wedge} 2, \Delta \mathrm{y}$ and $\Delta \mathrm{x}$ shows the distances from one dot to another from $\mathrm{x}$ and $\mathrm{y}$ axis, $\mathrm{M}$ stands for magnification and $\mathrm{t}$ stands for distances of each section from one another.

\section{Optical dissector method}

For counting numbers of neurons and neuroglia optical dissector method was used. Also microcator and counting frames were used. For calculating these numbers, sections were chosen by systematic uniform random sampling method. Thus 10 section was chosen. Then, all of the visibility of each section were examined using a microscope and with obj40. Based on counting on counting frames method, neurons were choosing which their nucleus were in the counting frame or they were on acceptable lines. Cases which were in contact with prohibited lines were unaccepted and they were not counted. For statistical analysis of data SPSS software (version 22) was used.

\section{Statistics}

In order to determine the significance of the difference between the experimental group, one-way ANOVA analysis method, and for comparison of differences between groups LSD test was used. In all comparisons, $\mathrm{P}<0.05$ level was considered as the criterion of significant difference (main \pm SEM).
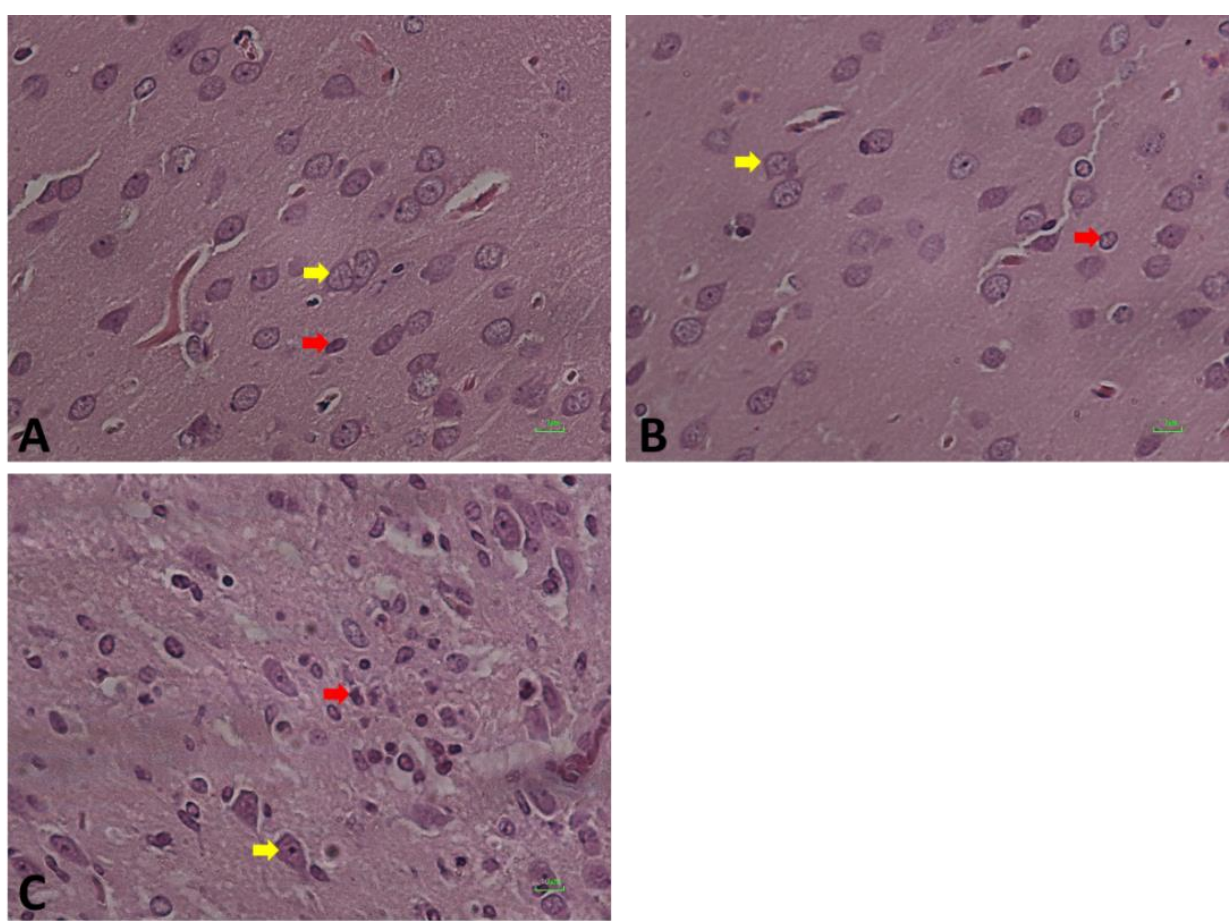

Figure 1. Prefrontal cortex of rat stained with $H \& E$ in different groups. A is control group, B is sham group and C is BPA group. Yellow arrows show neurons and red arrows show neuroglia.

\section{Results}

In this study H\&E staining was used to evaluate the density of nerve cells in the prefrontal region (Fig1. A,B,C). Data were extracted using stereology techniques and analyzed by ANOVA statistical methods.

\section{Total number of neurons}

Data are reported as mean. Total cell number estimates for the neocortical regions are shown in Figure 2. The mean number of neurons was 194.66 for BPA group and 367.99 for control group. There was a significant difference in the number of prefrontal frontal cortex neurons between BPA group and the control group ( $<<0.02$ ) (Fig2.A). Therefore, in this study the reduction of neuron numbers was observed in BPA group.

\section{Total number of neuroglia}

The mean number of neuroglia was 0.28 and 16.21 BPA a control group respectively. The results showed that the total number of the glial cells in the PFC was significantly increased (28.7) in the BPA group in comparison to the sham and control group $(\mathrm{p}<0.01)$ (Fig2.B).

\section{Volume of prefrontal cortex}

Average volume of prefrontal cortex in control group was 0.28 on the other hand the average volume of prefrontal cortex in BPA group was 0.23 (Fig2.C).

The study findings showed reduction in the volume of the prefrontal region in the BPA in comparison to the control group and there was significantly difference between these groups $(p<0.03)$. 


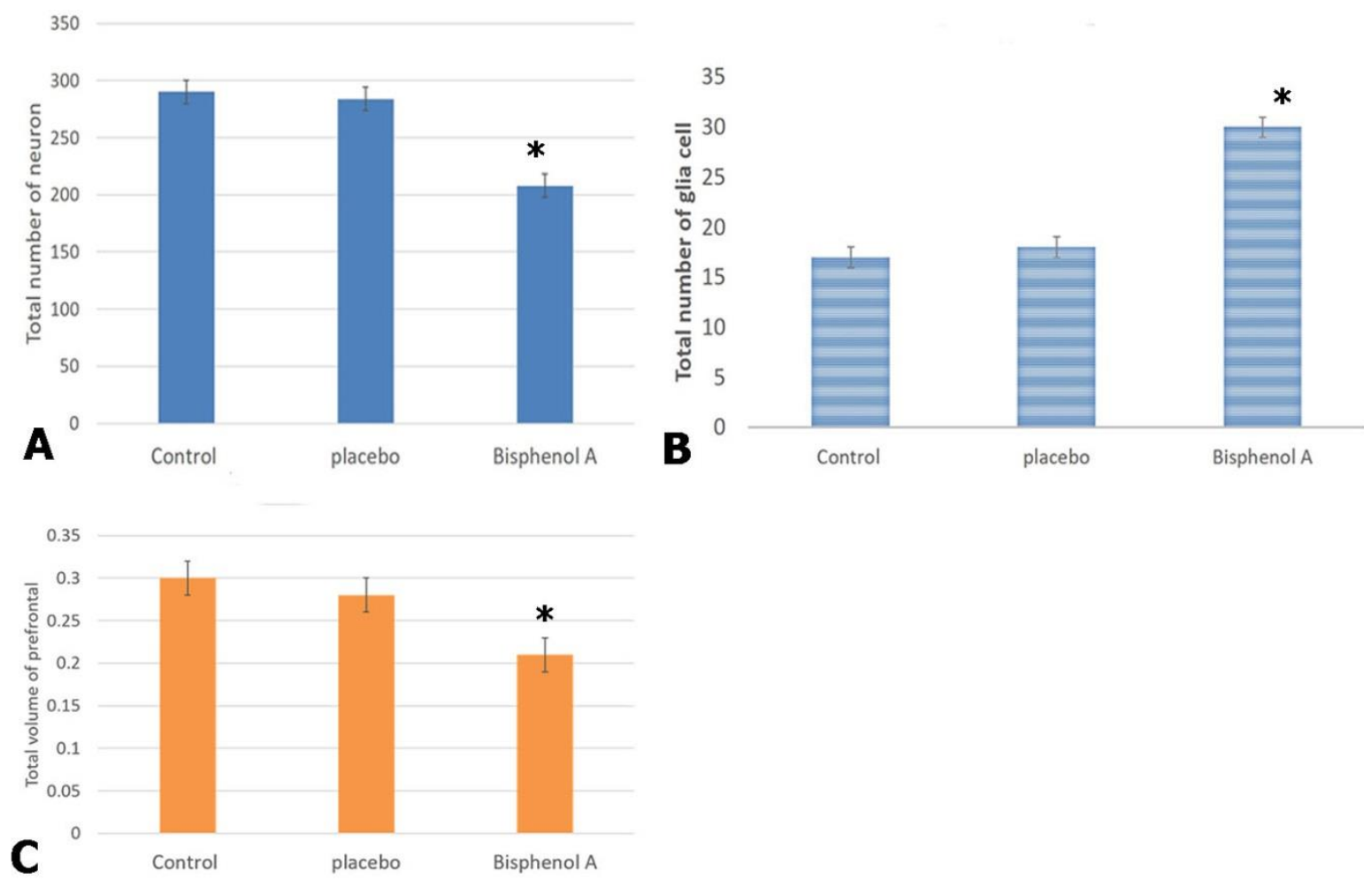

Figure2. Total number of neurons in different studying groups (A). Density of Neuroglia in different studying groupS (B). Prefrontal cortex volume in different groups $(\mathrm{C}){ }^{*}$; significantly different with both placebo and control group.

\section{Discussion}

The present study investigated the changes in the total volume and total number of the neurons and glial cells in PFC after BPA injection by using stereological methods. Due to the increase in environmental pollutants in the modern world and their destructive role on the nervous system's growth and development system, in this study we examined and analysed the destructive effects of BPA on the prefrontal area of male rats. The results of our observations showed that the injection of BPA reduces the number of neurons, reduces the volume of the prefrontal region, and also increases the number of glial cells. The present study also indicated that exposure to BPA reduced the number of the neurons in the $\mathrm{mPFC}$, which is in agreement with the results of another study conducted on the issue. In the present study, the number of glial cells in the mPFC was also quantified using stereological techniques and the results showed increasing in the number of the glial cells in the PFC after injection of BPA. In this study we have seen gliosis in response to damage to the prefrontal cortex. The number of neuroglia cell increased due to proliferation or hypertrophy of several different types of glial cells, including astrocytes, microglia, and oligodendrocytes.

The reduction of neuroglia number was predictable because after injection of BPA cause damage to neuron cell due to degeneration, and they make smaller volume of prefrontal area in the damaged area. an scar tissue. Which was similar to the results of other studies in this field (1518). Previous studies shown that that BPA in dosedependent behaviour increases oxidative stress levels, and lipid peroxidation, as well as DNA damage in cells and induces genotoxic effects in cells (19). BPA causes mitochondrial damage, resulting in producing less energy and more ROS, and oxidative stress occurs due to an imbalance between free radicals and antioxidants. Numerous reports suggest that BPA could cause programmed death in many cells, both in living organisms and in the laboratory.(2). BPA also alters the pattern of expression of some genes, especially by increasing the level of expression of Bax apoptotic gene and reducing the level of expression of $\mathrm{Bcl}-2$ antipyptoptychic gene. Increasing the expression of the Bax gene activates the gene waterfall and apoptotic signals, which are eventually transferred to the mitochondria and increase the activity of Caspases 3, 8, and 9. As a result changes in the rate of expression and activity of Caspases occurs which play an effective role in promoting the onset of apoptosis (24).

Studies conducted by Rahimi et al. showed that placing exposure of BPA to mice at doses of 10 and 50 micrograms per kilogram does not significantly changes liver's tissue structure. While higher doses (100 micrograms per kilogram) cause the release of inflammatory factors and the 
accumulation of macrophages in the liver tissue, it significantly increases the serum concentrations of AST and ALT enzymes(20). In addition, Eilam et al. demonstrate that exposure to 40 micrograms of BPA BPA reduced spatial memory in rats $(21)$. Wise investigated the effect of different doses of BPA in male and adolescent female mice after puberty. The results showed that the number of neurons did not change after puberty, but the number of glial cells in male mice decreased and increased in female mice (22). Observations from the present study showed that the volume and number of neurons decreased and the number of glial cells increased, which did not support Wise's results. The results of this study support the observations of Castro et al. on the changes that occurred in the prefrontal cortex and the possibility of pathophysiological disorders (23).

\section{Conclusion}

In the present study, more empirical evidence suggests that exposure to BPA reduces the number of peritoneal cortical neurons, increases the number of glial cells, and reduces the total volume of the prefrontal region, which can eventually lead to neurological damage and developmental disorders. Finally, given the ability of BPA to cause neurotoxicity in the developing brain, every effort should be made for eliminating this unnecessary element from many medical products, disposable utensils, mineral water bottles, PVC and baby food jars, or increasing the use of substances that can reduce or eliminate its toxic effects.

\section{Financial disclosure}

The authors declared no financial interest.

\section{Funding/Support}

This work is a part of the student's thesis of Habibi at Faculty of Medicine, Tehran Medical Sciences, Islamic Azad University, Tehran, Iran. The author(s) received no funding for this work.

\section{References}

1. Mandel ND, Gamboa-Loira B, Cebrián ME, Mérida-Ortega Á, López-Carrillo L. Challenges to regulate products containing bisphenol A: Implications for policy. salud pública de méxico. 2019;61(5, sep-oct):692-7.

2. Kimber I. Bisphenol A and immunotoxic potential: A commentary. Regulatory Toxicology and Pharmacology. 2017;90:358-63.

3. Cabado AG, Aldea S, Porro C, Ojea G, Lago J, Sobrado C, et al. Migration of BADGE (bisphenol A diglycidyl-ether) and BFDGE (bisphenol F diglycidyl-ether) in canned seafood. Food and chemical toxicology. 2008;46(5):1674-80.

4. Wazir U, Mokbel K. Bisphenol A: A concise review of literature and a discussion of health and regulatory implications. in vivo. 2019;33(5):1421-3.
5. Akash MSH, Sabir S, Rehman K. Bisphenol A-induced metabolic disorders: From exposure to mechanism of action. Environmental Toxicology and Pharmacology. 2020;77:103373.

6. Valentino R, D’Esposito V, Ariemma F, Cimmino I, Beguinot F, Formisano P. Bisphenol A environmental exposure and the detrimental effects on human metabolic health: is it necessary to revise the risk assessment in vulnerable population? Journal of endocrinological investigation. 2016;39(3):259-63.

7. Vahedi M, Saeedi A, Poorbaghi SL, Sepehrimanesh M, Fattahi M. Metabolic and endocrine effects of bisphenol A exposure in market seller women with polycystic ovary syndrome. Environmental Science and Pollution Research. 2016;23(23):23546-50.

8. Radwan M, Wielgomas B, Dziewirska E, Radwan P, Kałużny P, Klimowska A, et al. Urinary bisphenol A levels and male fertility. American journal of men's health. 2018;12(6):214451.

9. Murata M, Kang J-H. Bisphenol A (BPA) and cell signaling pathways. Biotechnology Advances. 2018;36(1):311-27.

10. Koike E, Yanagisawa R, Win-Shwe T-T, Takano H. Exposure to low-dose bisphenol A during the juvenile period of development disrupts the immune system and aggravates allergic airway inflammation in mice. International journal of immunopathology and pharmacology. 2018;32: 2058738418774897.

11. Fujiwara Y, Miyazaki W, Koibuchi N, Katoh T. The effects of low-dose bisphenol $\mathrm{A}$ and bisphenol $\mathrm{F}$ on neural differentiation of a fetal brain-derived neural progenitor cell line. Frontiers in Endocrinology. 2018;9:24.

12. Heidbreder CA, Groenewegen HJ. The medial prefrontal cortex in the rat: evidence for a dorso-ventral distinction based upon functional and anatomical characteristics. Neuroscience \& Biobehavioral Reviews. 2003;27(6):555-79.

13. Carlén M. What constitutes the prefrontal cortex? Science. 2017;358(6362):478-82.

14. Gundersen H, BENDTSEN TF, KORBO L, MARCUSSEN N, Møller A, Nielsen K, et al. Some new, simple and efficient stereological methods and their use in pathological research and diagnosis. Apmis. 1988;96(1- 6):379-94.

15. Elsworth JD, Jentsch JD, VandeVoort CA, Roth RH, Redmond Jr DE, Leranth C. Prenatal exposure to bisphenol A impacts midbrain dopamine neurons and hippocampal spine synapses in non-human primates. Neurotoxicology. 2013;35:113-20.

16. Eilam-Stock T, Serrano P, Frankfurt M, Luine V. BisphenolA impairs memory and reduces dendritic spine density in adult male rats. Behavioral neuroscience. 2012;126(1):175.

17. Asgari Hasanluyi E, Banan Khojasteh M, Fazeli M, Hatami H. Investigating the Neurotoxicity of Bisphenol A on Spatial Learning and Memory in Male Rats. Journal of Mazandaran University of Medical Sciences. 2016;26(140):192-6.

18. Kim ME, Park HR, Gong EJ, Choi SY, Kim HS, Lee J. Exposure to bisphenol A appears to impair hippocampal neurogenesis and spatial learning and memory. Food and chemical toxicology. 2011;49(12):3383-9.

19. Bowman RE, Luine V, Weinstein SD, Khandaker H, DeWolf S, Frankfurt M. Bisphenol-A exposure during adolescence leads to enduring alterations in cognition and dendritic spine density in adult male and female rats. Hormones and behavior. 2015;69:89-97. 
20. Rahimi O, Farokhi F, Banan Khojasteh SM. The effect of bisphenol A on liver tissue structure and liver enzymes. Qom University of Medical Sciences Journal. 2016;9(12):1-7.

21. Bansal A, Rashid C, Xin F, Li C, Polyak E, Duemler A, et al. Sex-and dose-specific effects of maternal bisphenol A exposure on pancreatic islets of first-and second-generation adult mice offspring. Environmental health perspectives. 2017;125(9):097022.

22. Wise LM, Sadowski RN, Kim T, Willing J, Juraska JM. Long-term effects of adolescent exposure to bisphenol A on neuron and glia number in the rat prefrontal cortex: differences between the sexes and cell type. Neurotoxicology. 2016;53:186-92.

23. Castro B, Sánchez P, Torres JM, Ortega E. Bisphenol A, bisphenol $\mathrm{F}$ and bisphenol $\mathrm{S}$ affect differently $5 \alpha$-reductase expression and dopamine-serotonin systems in the prefrontal cortex of juvenile female rats. Environmental research. 2015;142:281-7.

24. Taherianfard M, Taci A. Effects of Bisphenol A and Learning on the Distribution of GABA A $\alpha 1$ Receptors in the Rat Hippocampus and Prefrontal Cortex. Neurophysiology. 2015;47(1):23-9. 